Conditional gene expression

\section{Intelligent designs}

M Gossen

Gene Therapy (2006) 13, 1251-1252. doi:10.1038/sj.gt.3302829;

published online 20 July 2006

A manuscript recently published in Nature Methods by the Trono and Aebischer labs introduces a new transcriptionally regulated, lentivirus-based transgene delivery system functional in cultured cells as well as in mammalian organisms. ${ }^{1}$ Its compact, modular design permits the incorporation of inducible promoters for RNA polymerase-II and -III (RNAPII/III), applicable for different approaches in gene expression and RNA interference (RNAi).

While one might argue that similar tools for gene delivery and expression exist, it is the increasing sophistication in the design of these tools that is remarkable. Due to the intrinsic complexity of the engineered biological systems used for transferring genes as well as for their conditional expression, such tools will never be foolproof. Nevertheless, with alternative methods on-hand, progress in this field will be judged more and more by the likelihood of succeeding when adopting a given system to tackle a specific problem. Put simply, not if it can work, but if it will work is the question.

Lentivectors such as those introduced by Szulc et al. ${ }^{1}$ may be part of the answer, also as potential tools for increasingly less distant applications in gene therapy. Such regimens ultimately aim at transgenemediated, therapeutically beneficial alterations of the transcriptional profile in a limited subset of cells in the human body. To this end, numerous strategies have been considered or demonstrated at the level of proofof-principle. Whatever the specific approach, whatever the specific target, it is obvious that interventions in the genetic homeostasis of a human being should ideally be reversible. If not possible on the level of the transgene itself, at least its expression should be conditional. While inevitably adding to the complexity of the experimental design, in the long-run this conditionality appears to be imperative for most applications, both for safety reasons as well as for quantitative control over transgene expression as adequate for the desired physiological response.

The most promising transgene control systems available rely on engineered transcription factors, controlled by small-molecule inducing agents. Among them, those controlled by tetracyclines (like doxycycline) gained particular popularity due to the favorable pharmacokinetic properties of the inducer as well as to the thermodynamic properties of the Escherichia coli derived functional elements. ${ }^{2}$

Adding to the success of this regulatory principle, the tetracycline-sensitive interaction of a Tet repressor (TetR) DNA binding moiety with its recognition sequences, is the fact that such systems come in three different flavors: relying on authentic repressors competing with the transcription machinery for promoter access, or relying on TetR fusion proteins binding to DNA outside the actual promoter sequence, actively up- or downregulating gene expression via fused eukaryotic transcriptional control domains. While all three transcription factors can mediate transgene control in tissue cultures, the latter two approaches are also routinely used for conditional gene expression in transgenic mammals, mostly employing TetR-transactivator fusion proteins stimulating transcription of engineered minimal promoters in a doxycycline-controlled fashion. ${ }^{2}$ So far this application almost exclusively employed RNAPII promoters, directing inducible expression of protein encoding genes but also microRNAs with embedded short hairpin RNA (shRNA) moieties to mediate RNAi ${ }^{3,4}$ Moreover, when properly modified such transcriptional activators are also expected to function with RNAPIII. ${ }^{5}$ In contrast, for the inducible downregulation of gene expression, TetR/transcriptional silencer fusion proteins are used. ${ }^{6,7}$ Several silencer domains are promiscuous for RNAPII and -III promoters and some of them seem to function by triggering locally confined epigenetic changes around their binding sites. ${ }^{8}$ Using such a transcriptional silencer fusion proteins (TetR-KRAB ${ }^{6,9}$ ), Szulc et al. ${ }^{1}$ show efficient doxycycline control over several different RNAPII promoters in tissue cultures, in xenotransplantation experiments and also after lentivector-mediated transgenesis in mice, getting by with a singlevector strategy (for advantages of lentiviral vector systems, see Wiznerowicz and Trono ${ }^{10}$ ). Interestingly, in various experimental settings, the authors could switch transgene expression $\mathrm{ON}$ and $\mathrm{OFF}$ repeatedly ('doxycycling'). However, if this gene regulation involves epigenetic modifications, one should not expect it to follow the same kinetics as for repressors or activators interacting directly with the transcription machinery. A notable exception to the conditionality of the approach was the irreversible manifestation of the transgene shutdown when expression was switched OFF during early stages of embryogenesis, likely due to epigenetic reprogramming in mouse development. ${ }^{11}$

The same single-lentivector design was also successfully employed for the regulated expression of shRNAs from RNAPIII promoters, both in vivo and in vitro. Given the continuously increasing interest in conditional RNAi, these and similar vectors are guaranteed considerable attention for gene function analysis as well as for gene therapy. Such applications typically aim at a functional knockdown of the target gene(s) and not necessarily at a quasi-knockout. Thus, efficacy of shRNA regulation will mostly be judged by its own phenotypic criteria and not by foldness of regulation counted in orders of magnitude, contributing to the perceived robustness of conditional RNAi. What makes the design of these lentivectors attractive for end-users of the technology is their limited number of genetic control elements, reflected by the single vector strategy, the use of multicistronic RNAs as well as the autoregulatory mode of control chosen for silencer expression by a negative feedback mechanism (Figure 1). However, one should always keep in mind that individual control over functional elements of rather complex systems can also be an asset for optimizing conditional gene expression, when required. Such 


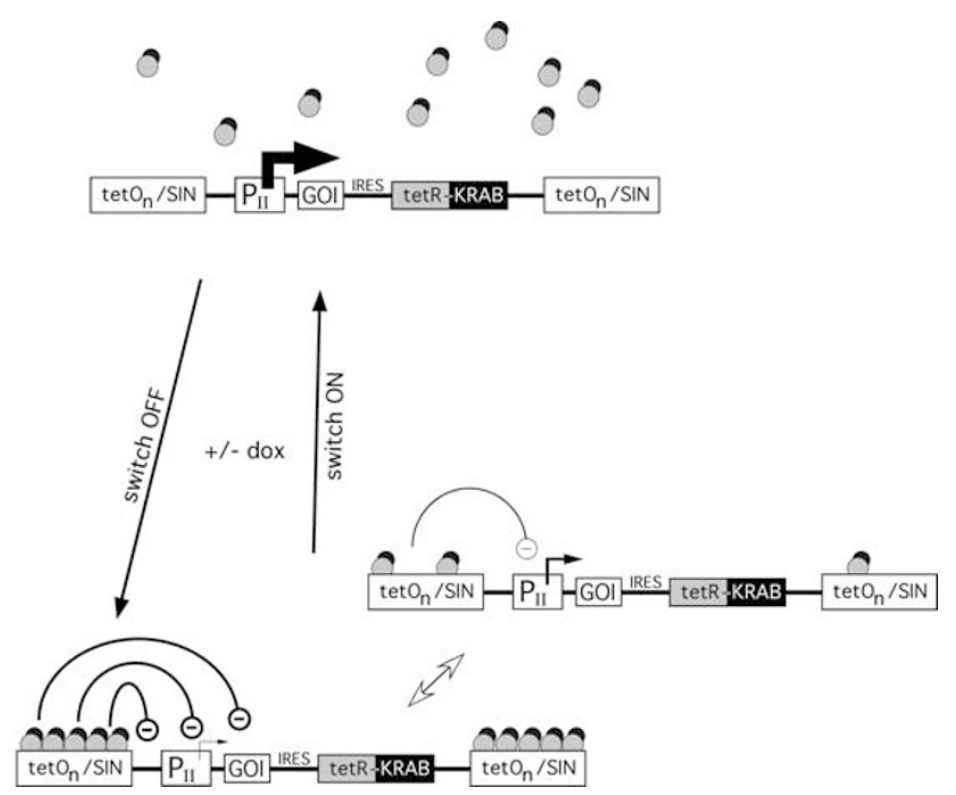

Figure 1 Conditional transgene expression by an autoregulatory TetR/silencer approach. Depicted is the basic lentivector design introduced by Szulc et al. ${ }^{1}$ Long terminal repeats (LTRs) used for vector construction are self-inactivating (SIN) and are combined with multiple tet operator sequences $\left(\right.$ tet $\left.\mathrm{O}_{\mathrm{n}}\right)$ serving as binding sites for TetR fusion proteins. These LTRs flank the central transcription unit of the lentivectors, comprised of (i) an RNAPII promoter $\left(\mathrm{P}_{\mathrm{II}}\right)$ directing transcription of (ii) a gene of interest (GOI), (iii) an internal ribosome entry site (IRES) allowing the bicistronic design with two open reading frames translated from one mRNA, and (iv) a tetR/transcriptional silencer fusion (tetR-KRAB; the corresponding protein is depicted by gray/black circles). The scheme does not discriminate between wild-type TetR and 'reverse' TetR fusions, ${ }^{2}$ both of which where used by Szulc et al. ${ }^{1}$ to either prevent or enable the DNA binding of those fusion proteins in response to doxycycline (dox) addition. What is referred to is the expression state, ON or OFF, mediated by those TetR fusions. The upper panel (ON) shows the state of maximal expression for the GOI (and thereby also tetR-KRAB). This state can be maintained as long as the dox conditions prevent TetR-KRAB from binding to the tet $\mathrm{O}_{\mathrm{n}} / \mathrm{SIN}$-type LTRs. Expression is switched OFF upon reversal of the dox conditions. Now TetR-KRAB, which is present at high intracellular concentrations, binds to the tetO sequences and shuts down transcription from $P_{\mathrm{II}}$. The repressive effect of the silencer proteins is only indicated for the $\mathrm{P}_{\mathrm{II}}$-proximal LTR $(-\Theta)$. Once $\mathrm{P}_{\mathrm{II}}$ is OFF, the concentration of the silencer protein will decrease and is expected to reach a point where repression is partially relieved. Resulting re-synthesis of TetR-KRAB will re-repress $P_{I I}$. This negative autoregulatory feedback loop is indicated by an open, double-headed arrow. As a consequence, the system is expected to hover up and down somewhat above the maximally repressed baseline level, at least when looking at the individual transcription unit. Conditional RNAi could be successfully added to this design by incorporating an RNAPIII promoter/shRNA-coding cassette in tetO ${ }_{n} /$ SIN, subjecting it to the repressive effect of TetR-KRAB. It will be interesting to learn if such an autoregulatory design is compatible with adjusting transgene expression to intermediate levels.

modifications should be possible though, including the use of activator systems for RNAPII driven miRNA/ shRNA hybrids as described., ${ }^{3,4}$ Right now it is too early to predict which approach for conditional RNAi will prevail for cellular systems or gene therapy. In contrast, when thinking about regulated shRNA expression in transgenic organisms, it is not trivial to achieve defined knockdown patterns by either repressors or silencers due to the problems in establishing the appropriate tissue distribution for such transcription factors as well as the lack of tissue-specificity of the RNAPIII promoters used. Strategies to overcome this limitation will inevitably result in an increase in experimental complexity. ${ }^{12}$ Thus, while including RNAi technology in lentivector-mediated transgenesis was rightfully heralded as a combo

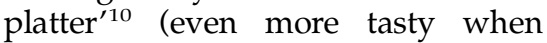
conditional), there is still room for dessert.
$M$ Gossen is at the Max-Delbrück-Center for Molecular Medicine, Robert-Rössle-Strasse 10, Berlin, Germany.

E-mail:mgossen@mdc-berlin.de

Published online 20 July 2006

1 Szulc J, Wiznerowicz M, Sauvain MO, Trono D, Aebischer P. A versatile tool for conditional gene expression and knockdown. Nat Methods 2006; 3: 109-116.

2 Gossen M, Bujard H. Studying gene function in eukaryotes by conditional gene inactivation. Annu Rev Genet 2002; 36: 153-173.

3 Dickins RA, Hemann MT, Zilfou JT, Simpson DR, Ibarra I, Hannon GJ et al. Probing tumor phenotypes using stable and regulated synthetic microRNA precursors. Nat Genet 2005; 37: 1289-1295.

4 Stegmeier F, Hu G, Rickles RJ, Hannon GJ, Elledge SJ. A lentiviral microRNA-based system for single-copy polymerase II-regulated RNA interference in mammalian cells. Proc Natl Acad Sci USA 2005; 102: 13212-13217.

5 Das G, Hinkley CS, Herr W. Basal promoter elements as a selective determinant of transcriptional activator function. Nature 1995; 374: 657-660.

6 Deuschle U, Meyer WK, Thiesen HJ. Tetracycline-reversible silencing of eukaryo- tic promoters. Mol Cell Biol 1995; 15: 1907-1914.

7 Freundlieb S, Schirra-Muller C, Bujard H. A tetracycline controlled activation/repression system with increased potential for gene transfer into mammalian cells. J Gene Med 1999; 1: 4-12.

8 Ayyanathan K, Lechner MS, Bell P, Maul GG, Schultz DC, Yamada Y et al. Regulated recruitment of HP1 to a euchromatic gene induces mitotically heritable, epigenetic gene silencing: a mammalian cell culture model of gene variegation. Genes Dev 2003; 17: 1855-1869.

9 Wiznerowicz M, Trono D. Conditional suppression of cellular genes: lentivirus vectormediated drug-inducible RNA interference. J Virol 2003; 77: 8957-8961.

10 Wiznerowicz M, Trono D. Harnessing HIV for therapy, basic research and biotechnology. Trends Biotechnol 2005; 23: 42-47.

11 Morgan HD, Santos F, Green K, Dean W, Reik W. Epigenetic reprogramming in mammals. Hum Mol Genet 2005; 14: R47-R58.

12 Ventura A, Meissner A, Dillon CP, McManus M, Sharp PA, Van Parijs L et al. Cre-loxregulated conditional RNA interference from transgenes. Proc Natl Acad Sci USA 2004; 101: 10380-10385. 